\title{
A note on numerical computations of large amplitude standing waves
}

\author{
By PHILIP G. SAFFMAN \\ California Institute of Technology, Pasadena, CA 91125 \\ AND HENRY C. YUEN \\ TRW Defense and Space Systems Group, Redondo Beach, CA 90278
}

(Received 19 October 1978 and in revised form 12 February 1979)

Numerical solutions of the inviscid equations that describe standing waves of finite amplitude on deep water are reported. The calculations suggest that standing waves exist of steepness, height and energy greater than the limiting wave of Penney \& Price (1952). The computed profiles are found to be consistent with Taylor's (1953) experimental observations.

\section{Introduction}

The existence of a finite amplitude steady progressive deep water gravity wave of greatest height has been demonstrated (Stokes 1880; Michell 1893; Toland 1978). In contrast, the nature of large amplitude standing waves and the existence of a highest achievable profile are unsettled issues. Penney \& Price (1952) calculated approximate shapes of standing waves using a series expansion in wave amplitude to fifth order. By assuming that the downward acceleration at the crest of the wave of greatest height is $g$, they obtained a highest wave profile with $H / L=0 \cdot 218$, crest angle equal to $90^{\circ}$, total energy per unit area $E=0.0968 g(L / 2 \pi)^{2}$, and frequency of oscillation $\sigma=0.949 \sigma_{0}$, where $\sigma_{0}=(g L / 2 \pi)^{\frac{1}{2}}$ is the frequency of the infinitesimal wave, $L$ is the wavelength and $H$ is the peak-to-trough wave-height.

The validity of the arguments of Penney \& Price was questioned by Taylor (1953). However, the results of his experiments to determine the highest standing wave profile were found to be consistent with the contentions of Penney \& Price.

We have performed a series of calculations of standing waves using a numerical scheme based on that developed by Longuet-Higgins \& Cokelet (1976) which solves the exact free surface unsteady flow problem of an inviscid, irrotational, incompressible fluid with periodic boundary conditions for prescribed initial conditions and external pressure. Our calculations show the existence of standing waves with $H / L$ greater than $0 \cdot 218$, maximum local slope exceeding $45^{\circ}$, and $E$ larger than $0.0968 g(L / 2 \pi)^{2}$. The computed profiles are found to be also consistent with Taylor's experiment.

\section{Numerical results}

Sixty spatial points were used in our calculations. A fifth order polynomial smoothing scheme was needed to stabilize the computation. The accuracy of the solutions 
was monitored by computing the mass flux $\Omega$ across the surface, which should be zero. In all our results, the value of $\Omega$ oscillated in time with an absolute magnitude less than $0 \cdot 0001$. We chose units such that $g=1, L=2 \pi, \sigma_{0}=1$ and the infinitesimal wave period is $2 \pi$. We first show results for waves generated by the application of an external pressure for a finite period of time to an initially flat stationary surface, after which the wave is allowed to oscillate freely. The waves generated in such a manner have an identifiable dominant frequency, but like those in a physical experiment do not repeat exactly and are not 'pure' standing waves. We used the pressure distribution:

$$
p(x, t)=\left\{\begin{array}{ll}
p_{0} \cos x \sin t & 0<t<t_{0} \\
0 & t>t_{0}
\end{array}\right\}
$$

with different values of $p_{0}$ and $t_{0}$. We calculated the wave profiles $\eta(x, t)$, the per unit area potential energy $V$, kinetic energy $T$, total energy $E=T+V$, wave steepness $H / L$, maximum slope $\alpha$ over the wave profile, the acceleration at the crest and at the trough, and the frequency of oscillation $\sigma$. Results for representative cases are as follows.

Case 1. $p_{0}=0 \cdot 1, t_{0}=\pi$. The total energy rose from zero to 0.00615 at $t=\pi$ and remained constant thereafter (this provided a check on the accuracy of the calculation). When the wave went free, $T=0$ and $H / L=0.05$. For $t>t_{0}$, the kinetic and potential energies oscillated with a frequency indistinguishable from $2 \sigma_{0}$. The exchange of energy between $T$ and $V$ was only almost complete: $V$ was not exactly zero when $T$ was a maximum, which indicates that the surface was never exactly flat. The profile oscillated with dominant frequency $\sigma_{0}$, but did not repeat exactly after each period.

Case 2. $p_{0}=0.3, t_{0}=\pi$. The results were similar to case 1 , with $E=0.0545$ and $H / L=0.15$ at $t=\pi$, except that the frequency $\sigma$ is now $1 \cdot 002 \sigma_{0}$ owing to effects of finite amplitude.

Case 3. $p_{0}=0 \cdot 3, t_{0}=1.5 \pi$. In this case, the computation failed at $t=3 \cdot 36 \pi$, after approximately 1.5 half-periods of free oscillation. When the wave went free, almost all the energy was kinetic and $E=0 \cdot 1247$, after which the kinetic and potential energies oscillated periodically with period $1.079 \pi$ throughout the computation. The minimum values of $V$ and $T$ were close to zero, indicating as before an almost complete exchange of energy. The breakdown occurred as $H / L$ was decreasing after passing through its second maximum as a free wave, and was caused by coalescence of two points at the crest. The largest value of $H / L$ was 0.272 at $t=3.056 \pi$; the corresponding value of $\alpha$ (maximum slope) was $50^{\circ}$ and the downward acceleration at the crest was 0.97 . For $t>3.056 \pi, H / L$ decreased, the downward vertical acceleration approached unity, and the angle $\alpha$ continued to increase. At breakdown, when $H / L=0 \cdot 245$, the vertical acceleration became unity and the crest appeared cusped with $\alpha$ close to $60^{\circ}$. Figure 1 shows a composite time plot of the wave profile $\eta(x, t)$.

Case 4. $p_{0}=0 \cdot 28, t_{0}=1 \cdot 5 \pi$. The input conditions were the same as case 3 , except that the pressure was slightly smaller. The value of $E$ in the free wave was $0 \cdot 109$. In this case, the computation was continued until $t=6 \pi$, and no breakdown occurred. The half-period was $1 \cdot 063 \pi$. $T$ and $V$ were closely periodic. $H / L$ oscillated with the same frequency but with a $20 \%$ variation in amplitude. The computed maximum value of $H / L$ was $0 \cdot 258$ at $t=3 \pi$; the corresponding value of $\alpha$ was $52^{\circ}$. The maximum value of $\alpha$ was $54^{\circ}$, at $t=3 \cdot 25 \pi$ and $H / L=0 \cdot 21$. The maximum vertical acceleration 


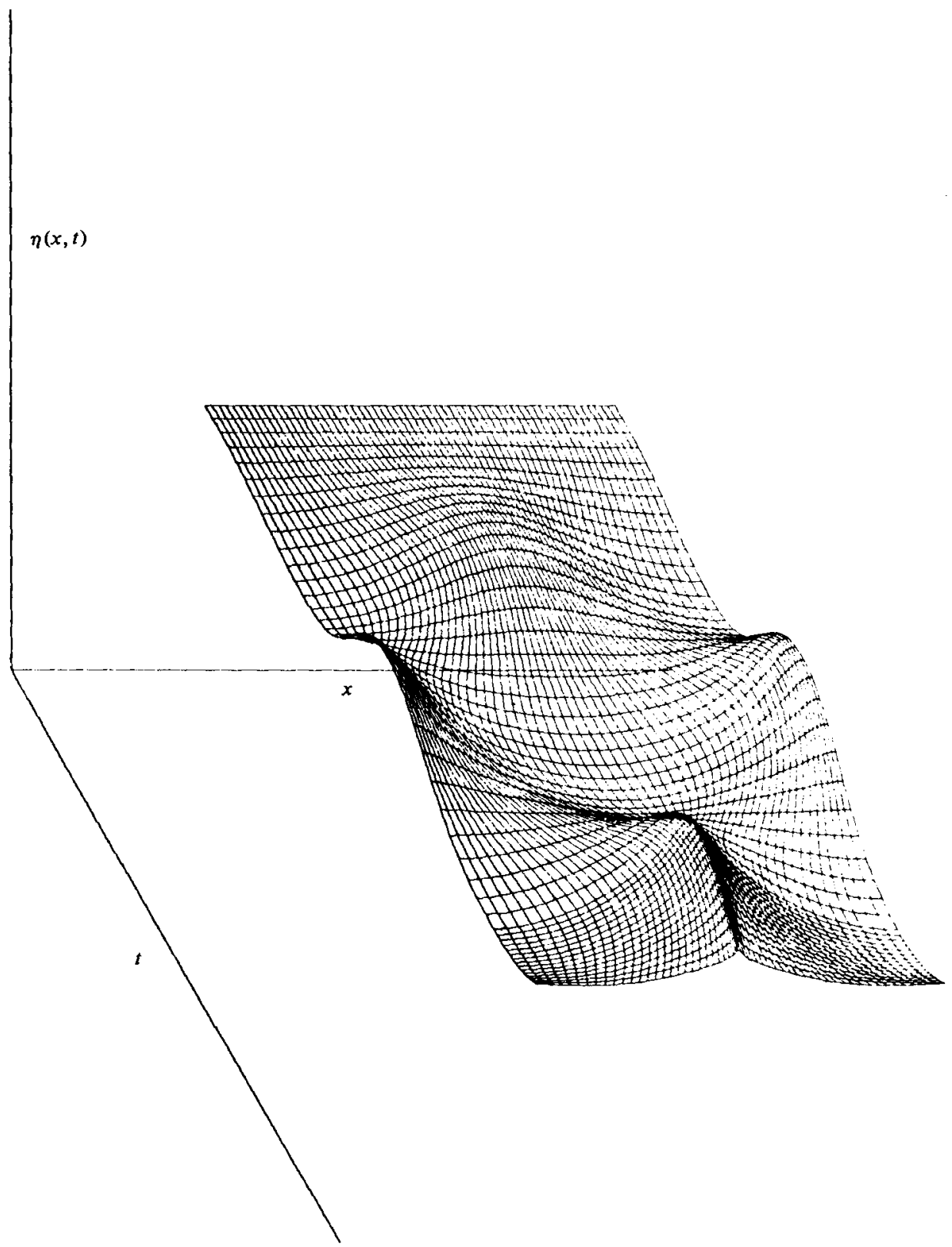

Figure 1. Wave profile $\eta(x, t)$ of a standing wave generated from a flat surface by an external pressure with $p_{0}=0 \cdot 30$ (case 3). Scales in plot: $-6 \cdot 0 \leqslant x \leqslant 4 \cdot 0,0 \leqslant t \leqslant 11 \cdot 03$, $-4 \cdot 0 \leqslant \eta \leqslant 6 \cdot 0$.

computed was 0.97 at $t=3.596 \pi$. Figure 2 shows time plots of wave profile, $H / L$ and $\alpha$. The highest and sharpest profiles are shown later in figure 4 compared with Taylor's experimental data.

We now describe results of waves generated by releasing an elevated body of water from rest. The initial profiles were the approximate five mode solution given by Penney 


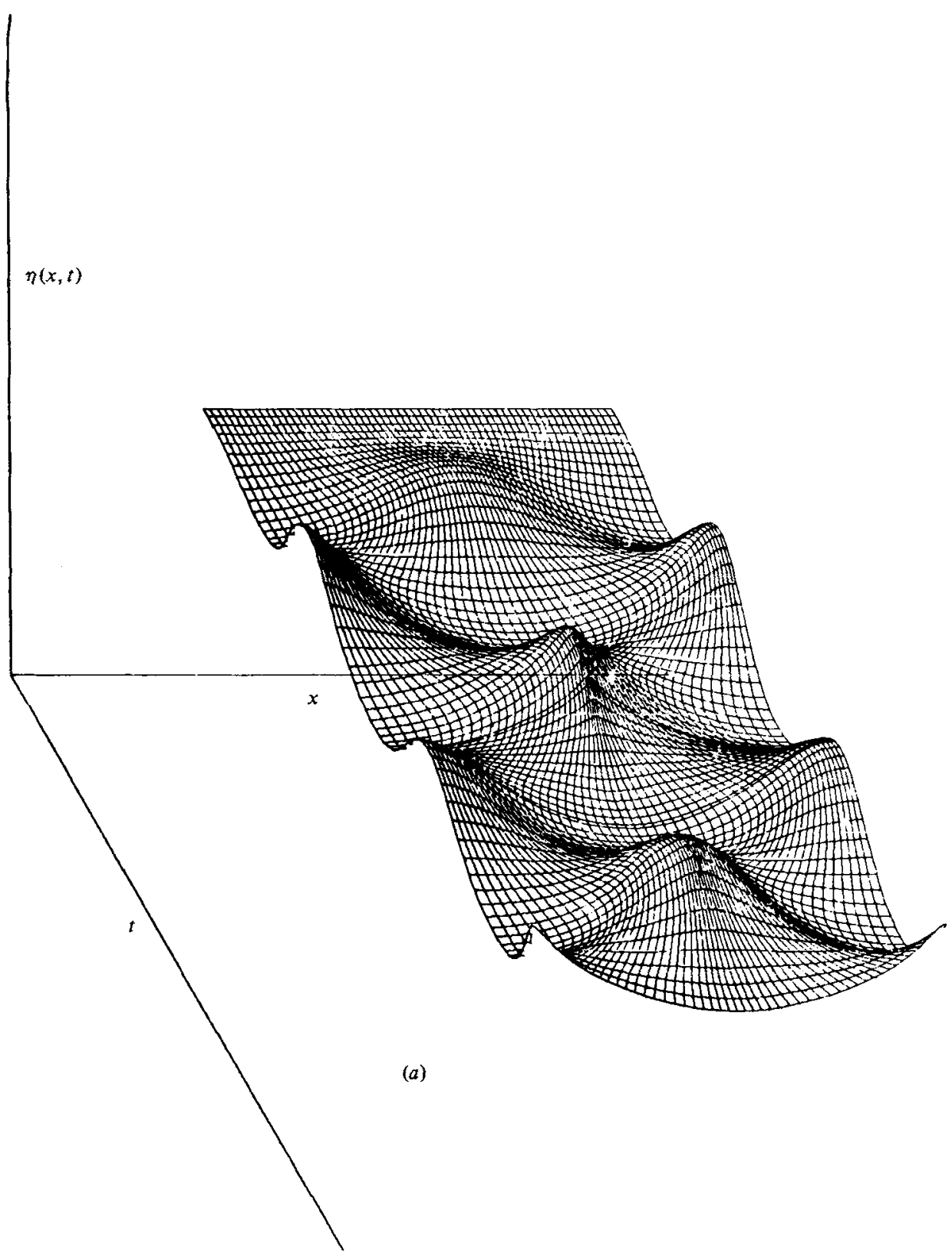

Figure $2(a)$. For legend see p. 711.

$\&$ Price taken at the time when the wave is instantaneously at rest. The magnitude of the wave is characterized by Penney \& Price's parameter $A$, roughly proportional to the amplitude of the first harmonic. The error in Penney \& Price's approximate solution is reflected in the degree of departure of the computed wave from a pure standing wave.

Case 5. $A=0.52$. This value of $A$ has special significance. For if instead of maximizing $H / L$ subject to the constraint that the downward acceleration at the crest does 

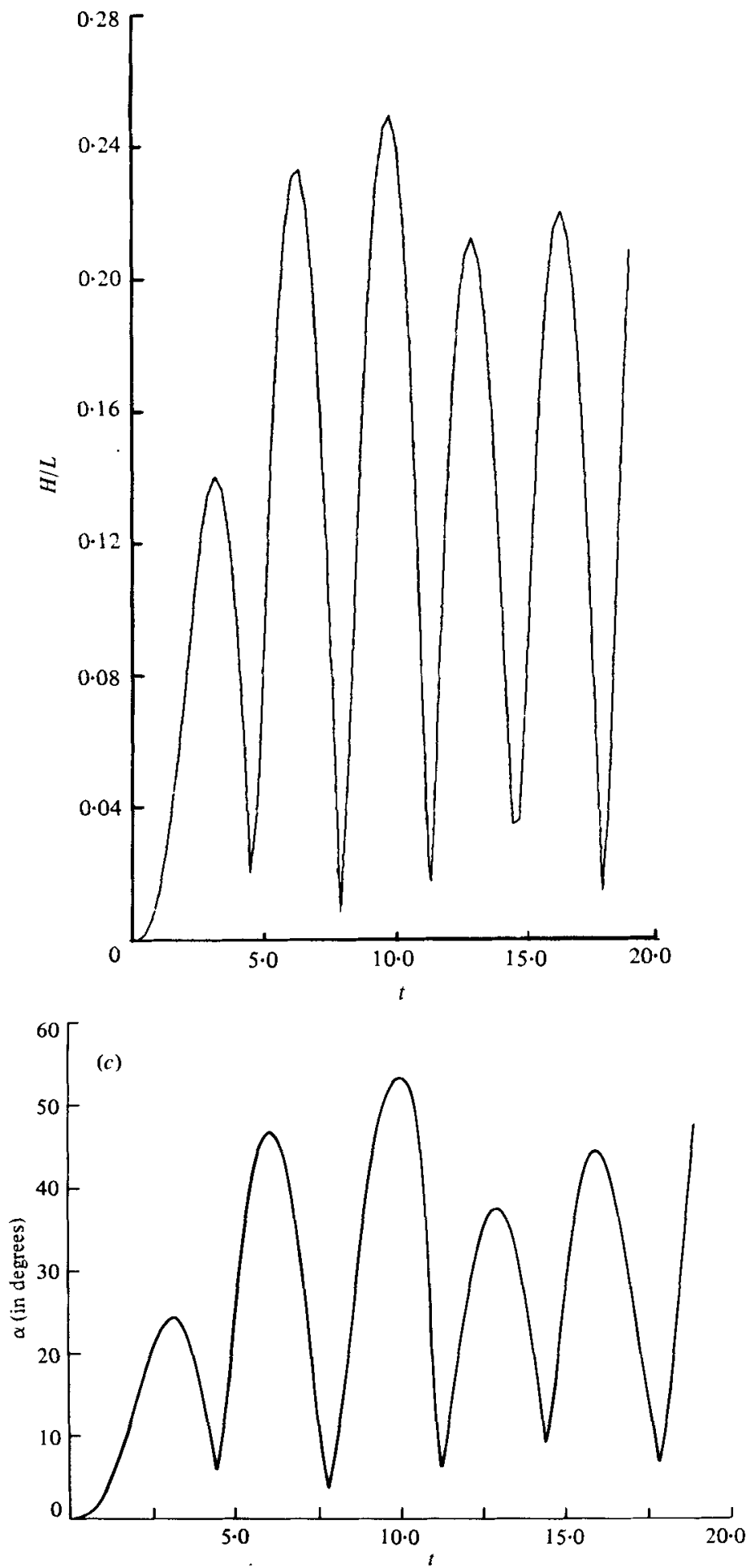

FigUre 2. Numerical results for a standing wave generated from a flat surface by an external pressure with $p_{0}=0.28$ (case 4). (a) Wave profile $\eta(x, t)$, scales in plot: $-6 \cdot 0 \leqslant x \leqslant 4 \cdot 0$, $0 \leqslant t \leqslant 18 \cdot 85,-4.0 \leqslant \eta \leqslant 6 \cdot 0 ;(b) H / L$ as a function of $t ;(c)$ slope $\alpha$ (in degrees) as a function of $t$. 


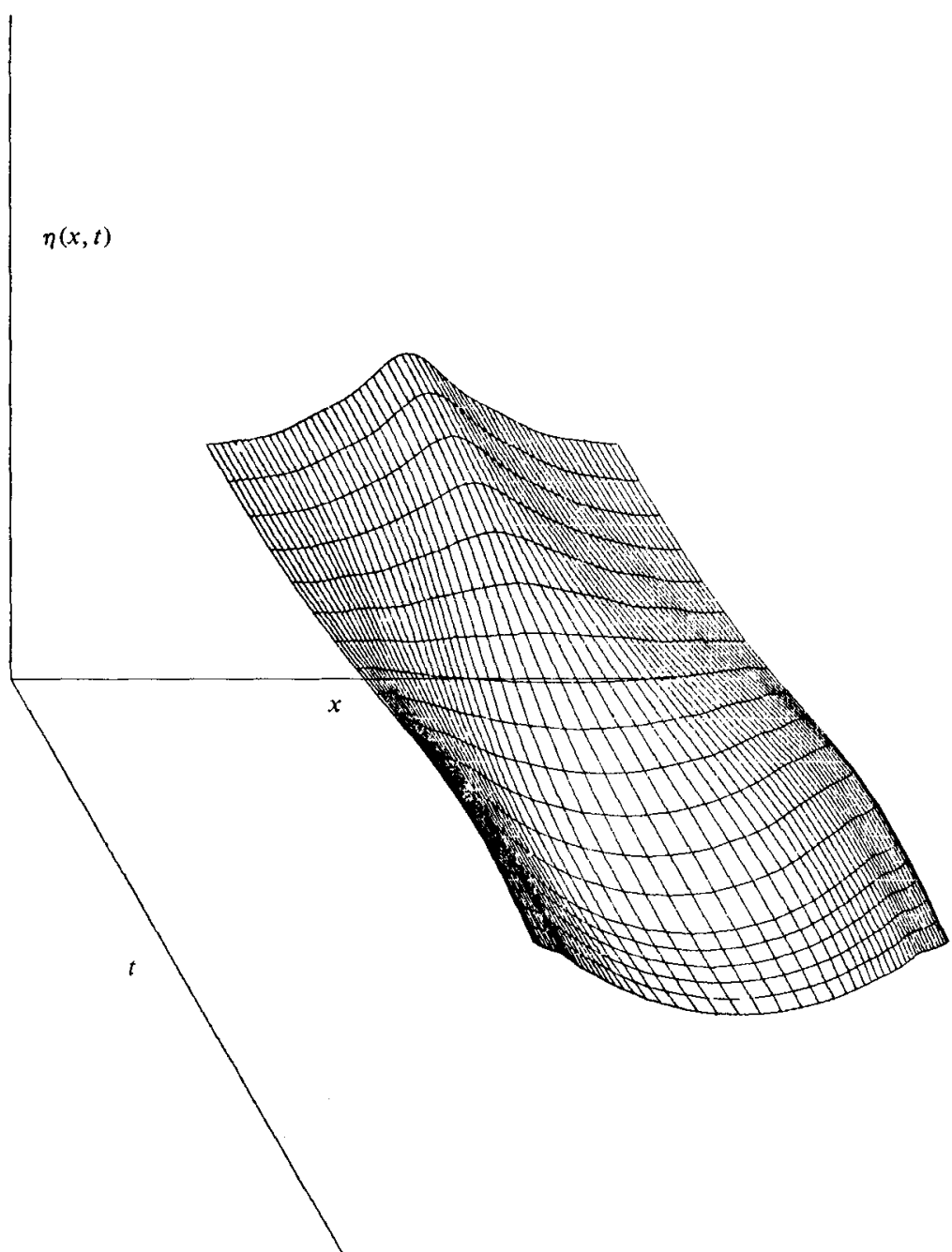

Figure 3. Computed wave profile $\eta(x, t)$ using the approximate profile of Penney \& Price (1952) with $A=0.592$ as initial condition (case 6). Scales in plot: $-6.0 \leqslant x \leqslant 4.0,0 \leqslant t \leqslant 4.0$, $-4 \cdot 0 \leqslant \eta \leqslant 6 \cdot 0$. Note that the crest of the wave at the time of breakdown is located at the edges of the plot.

not exceed $g$ (which gives $A=0.592$ and $H / L=0.218$ ), it is required that $\partial p / \partial y=0$ at the crest (the condition $\partial p / \partial y=0$ was used to derive the $90^{\circ}$ crest angle), then $A$ is found to be 0.52 . If the solution were exact, the values of $A$ obtained both ways would have been the same. According to Penney \& Price's formulae, the corresponding half-period would be $1.040 \pi$, with $H / L=0.185$ and $E=0.0731$.

The computed wave profiles oscillated smoothly, with the exchange of energy between $V$ and $T$ being almost complete. The half-period obtained from the oscillations of $V$ and $T$ was $1.036 \pi$. The wave profiles were almost, but not perfectly periodic. The value of $H / L$ had a $1.3 \%$ variation about a mean value of $0.184(H / L=0.185$ at $t=0,0.186$ at $t=1.036 \pi$, and 0.182 at $t=2 \cdot 072 \pi)$. The maximum slope $\alpha$ had a 


\begin{tabular}{|c|c|c|c|c|c|}
\hline Case & $E$ & Half-period & $(H / L)_{\max }$ & $\alpha_{\max }$ & $(a c c)_{\max }$ \\
\hline \multicolumn{6}{|c|}{ Non-breaking cases } \\
\hline 1 & 0.00615 & $\pi$ & 0.05 & $10^{\circ}$ & $0 \cdot 18$ \\
\hline 2 & 0.0545 & $1 \cdot 002 \pi$ & $0 \cdot 15$ & $31 \cdot 4^{\circ}$ & 0.52 \\
\hline \multirow[t]{2}{*}{5} & 0.0731 & $1 \cdot 036 \pi$ & $0 \cdot 186$ & $36 \cdot 4^{\circ}$ & 0.870 \\
\hline & & $(1 \cdot 040 \pi)$ & $(0 \cdot 185)$ & $\left(36 \cdot 4^{\circ}\right)$ & $(0.750)$ \\
\hline 4 & $0 \cdot 109$ & $1.063 \pi$ & 0.258 & $54^{\circ}$ & 0.97 \\
\hline \multicolumn{6}{|c|}{ Breaking cases } \\
\hline \multirow[t]{2}{*}{6} & 0.0968 & $1 \cdot 050 \pi$ & $0 \cdot 222$ & $67^{\circ *}$ & $1.05^{*}$ \\
\hline & & $(1 \cdot 053 \pi)$ & $(0 \cdot 218)$ & $\left(46^{\circ}\right)$ & $(1 \cdot 0)$ \\
\hline \multirow[t]{2}{*}{7} & $0 \cdot 119$ & $1.066 \pi$ & $0 \cdot 285$ & $85^{\circ} *$ & $1.0^{*}$ \\
\hline & & $(1 \cdot 066 \pi)$ & $(0 \cdot 247)$ & $\left(59^{\circ}\right)$ & $(1 \cdot 147)$ \\
\hline 3 & 0.1247 & $1.079 \pi$ & 0.272 & $50^{\circ} *$ & $1.0^{*}$ \\
\hline
\end{tabular}

( ) The corresponding results calculated from formulae given by Penney \& Price (1952).

* Denotes that the value is obtained from the last numerical output before computational breakdown.

TABLE 1. Summary of computational results.

$6.5 \%$ variation about a mean value of $34.7^{\circ}\left(\alpha=36 \cdot 4^{\circ}\right.$ at $t=0,35 \cdot 3^{\circ}$ at $t=1 \cdot 036 \pi$, and $32.5^{\circ}$ at $t=2.072 \pi$ ). The downward crest acceleration had a variation of $11 \%$ about a mean value of 0.784 (being 0.75 at $t=0,0.87$ at $t=1.036 \pi$ and 0.733 at $t=2 \cdot 072 \pi$ ). Comparison of the computed results with Penney \& Price's five mode expression shows good agreement.

Case 6. $A=0 \cdot 592$. According to Penney \& Price, this approximates the pure standing wave of maximum height. For this value of $A$, the Penney \& Price halfperiod is $1.056 \pi, H / L$ is $0 \cdot 218, E$ is 0.0968 , the initial downward acceleration at the crest is equal to unity and the maximum slope $\alpha$ is close to $45^{\circ}$. The computed profiles, however, broke down at $t=1 \cdot 273 \pi$. At breakdown, the wave was on its way down after reaching the second maximum at $t=1 \cdot 082 \pi$ with $H / L=0.222$ : The value of $\alpha$ was $61 \cdot 5^{\circ}$. The maximum computed value for $\alpha$ was $67^{\circ}$, achieved at $t=1 \cdot 273 \pi$, with $H / L=0 \cdot 174$. The profile at the time of breakdown possessed a sharp protrusion at the tip of the crest, qualitatively resembling the experimental profiles obtained by Taylor (1953, figures 14 to 20 ) for breaking standing waves (see figure 3 ). The halfperiod obtained from the oscillations of $V$ and $T$ was $1.050 \pi$, which indicated that the wave of maximum height was not the wave with maximum potential energy. The downward acceleration at the crest was 0.85 at $t=0,1.05$ at $t=1.082 \pi$, and changed sign abruptly as $t$ approached $1 \cdot 273 \pi$, suggesting that the calculation is not reliable beyond that time.

Case.7. $A=0 \cdot 65$. For this value of $A$, the downward crest acceleration according to Penney \& Price should be greater than $g$ and the wave should break on its way down. The computed wave, however, had an initial downward crest acceleration of 0.95 , and was able to complete one half-period before breakdown. From the oscillations of $V$ and $T$, the half-period was found to be $1 \cdot 066 \pi$. The computation broke down at $t=1 \cdot 166 \pi$. The maximum computed value for $H / L$ was 0.285 at $t=1.066 \pi$, the value of $\alpha$ at this time was $83^{\circ}$. The maximum value of $\alpha$ was $85^{\circ}$ achieved at the final time computed, with $H / L=\mathbf{0} \cdot 276$. The waveform at the time of breakdown again had a cusped protrusion at the crest. 

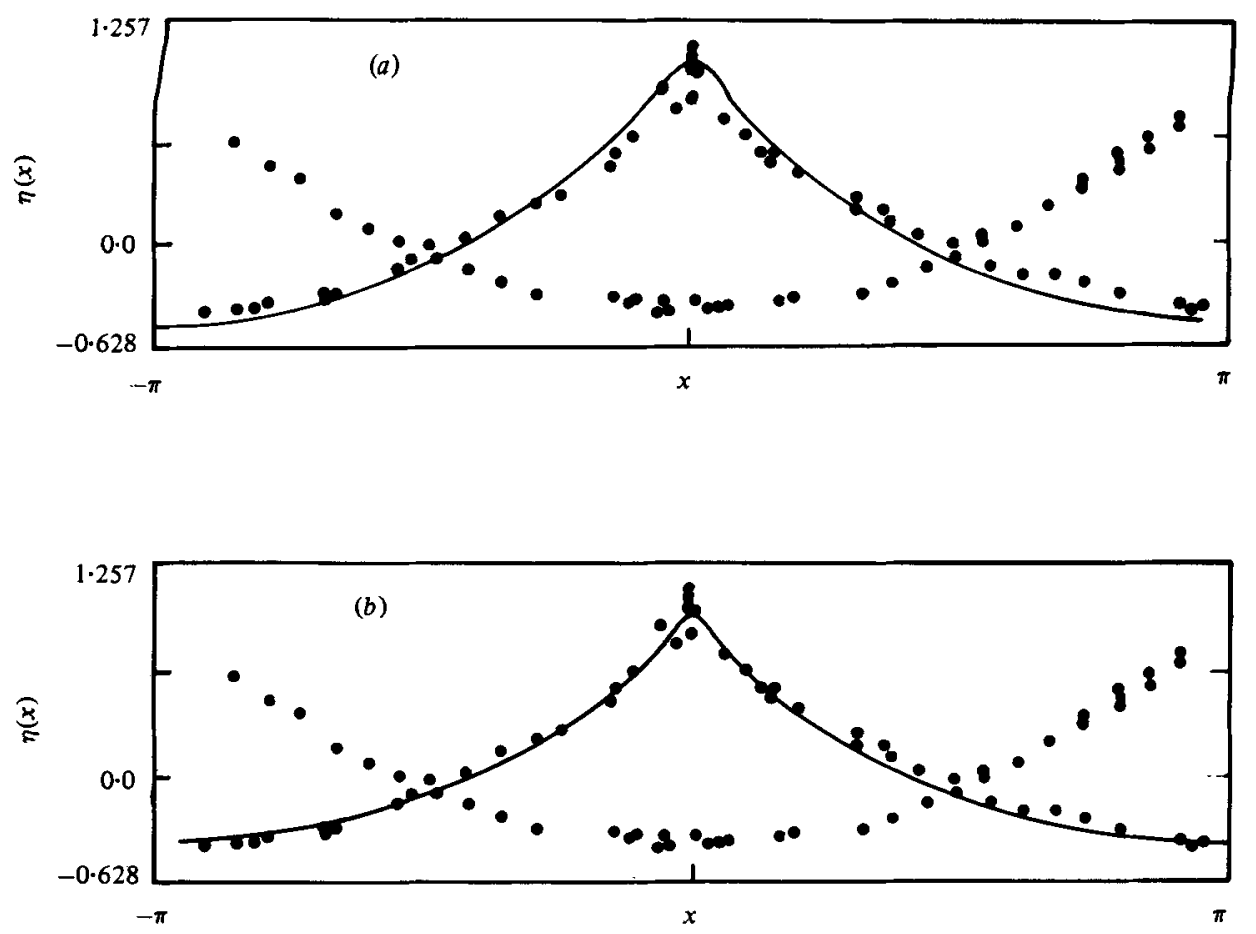

Figure 4. Comparison of numerical results with experimental data of Taylor (1953). (a) Experimental data compared to highest computed wave profile for case 4 (shown in figure 2); (b) experimental data compared to profile with maximum slope for case 4 (shown in figure 2). -

\section{Discussion}

Figure 4 shows a comparison of case 4 with the data of Taylor (1953). The numerical curves are those of greatest height and greatest slope that were computed. The agreement between computation and experiment seems as close as that between experiment and the Penney \& Price prediction. In addition, the appearance of a protuberance at the crest just before the wave breaks is in qualitative agreement with Taylor's observations, although the computations are strictly two-dimensional and cannot account for the ensuing three-dimensional behaviour.

The method of generation appears to affect the greatest height that a wave can achieve before breaking. For example, case 6 (release from elevated rest) describes a breaking wave with energy and height substantially less than that of a non-breaking wave (case 4) produced by application of pressure for a finite time.

Some of the important numbers of each case are listed in table 1. Provided the wave does not break, the calculated values of the half-period, maximum value of $H / L$, maximum slope, and maximum downward acceleration are found to be monotonic in $E$.It is to be particularly noted that we found standing waves of greater energy, slope, height and period than the limiting wave predicted by Penney \& Price. The numerical results do not support the argument for a $90^{\circ}$ crest on the wave of greatest height. The existence of breaking solutions, however, does suggest that there is an upper limit on the amplitude of standing waves. 


\section{REFERENCES}

Longuet-Higains, M. S. \& Cokelet, E. D. 1976 The deformation of steep surface waves on water. I. A numerical method of computation. Proc. Roy. Soc. A 350, 1-36.

Mrchell, J. H. 1893 The highest waves in water. Phil. Mag. 36 (5), 430-437.

Penney, W. G. \& Price, A. T. 1952 Finite periodic stationary gravity waves in a perfect liquid. Phil. Trans. Roy. Soc. A 244, 254-284.

Stokes, G. G. 1880 Considerations relative to the greatest height of oscillatory waves which can be propagated without change of form. Mathematical and Physical Papers, vol. I, pp. 225-238. Cambridge University Press. Also: Supplement to a paper on the theory of oscillatory waves. Mathematical and Physical Papers, vol. I, pp. 314-326.

TayLor, G. I. 1953 An experimental study of standing waves. Proc. Roy. Soc. A 218, 44-59.

Toland, J.F. 1978 On the existence of a wave of greatest height and Stokes's conjecture. Proc. Roy. Sac. A 363, 469-485. 